\title{
Acessibilidade dos equipamentos hoteleiros: perceções dos clientes
}

\section{Jenny Sousa ${ }^{a}$, Catarina Mangas ${ }^{\mathrm{b}}$}

aESECS, CICS.NOVA.IPLeiria-iACT, CI\&DEI, Politécnico de Leiria, Portugal, jenny.sousa@ipleiria.pt, ${ }^{b} E S E C S$, CICS.NOVA.IPLeiria-iACT, CI\&DEI, Politécnico de Leiria, Portugal, catarina.mangas@ipleiria.pt

\begin{abstract}
Resumo
A acessibilidade na prestação de serviços hoteleiros tem vindo a assumir uma importância cada vez maior, deixando de ser encarada como uma obrigação, mas antes como uma oportunidade de rentabilização e uma vantagem competitiva. Na verdade, as unidades hoteleiras têm consciência de que o segmento do mercado associado ao turismo acessivel está a crescer significativamente, salientando-se como uma oportunidade de negócio que não deve ser menosprezada. Conscientes de que a capacidade de atração dos lugares passa, cada vez mais, pela forma como estes podem ser utilizados por todos, os hotéis veem no conceito de acessibilidade transversal uma oportunidade de valorização. Este conceito, o de acessibilidade transversal, tem sido particularmente usado nas áreas do turismo/cultura e traduz-se na identificação de soluções que sejam úteis ao maior número de pessoas possivel, ao invés de adaptações específicas para determinado grupo de pessoas (com ou sem deficiência), que só sirvam alguns. Intimamente relacionado com o conceito de desenho universal, a acessibilidade transversal pode ser menos dispendiosa e mais lucrativa, por não implicar diversas soluções formuladas em função dos destinatários, mas apenas uma solução. Desta forma, é assegurado a todos os clientes um maior nível de autonomia na utilização das diversas valências da unidade hoteleira, no pressuposto de que quanto maior for o grau de independência, maior será a satisfação. Com este trabalho pretendemos discutir a importância da acessibilidade nos equipamentos hoteleiros e conhecer as soluções de acessibilidade mais indicadas em plataformas de apoio às pessoas que procuram espaços de alojamento inclusivos.
\end{abstract}

Palavras-chave: Turismo acessivel; Equipamentos hoteleiros acessiveis; Tur4all 


\section{Introdução}

O mercado do turismo acessível detém, cada vez mais, um lugar de destaque nas sociedades pós-modernas. Este tipo de turismo preconiza espaços e serviços capazes de responder às características de cada pessoa, independentemente das suas necessidades no momento pessoas com deficiência, pessoas idosas, famílias com crianças pequenas, grávidas, pessoas com a mobilidade temporariamente reduzida, entre outros - numa perspetiva de turismo para Todos.

Este paradigma tem um duplo objetivo e conjuga dois aspetos muito importantes: por um lado, vai ao encontro do direito das pessoas ao lazer e desejo de fazer turismo, portanto responde a uma questão social; por outro, ao responder às necessidades específicas de cada um, aposta-se num melhor serviço, refletindo-se no reforço da competitividade dos diversos equipamentos e locais, isto é, potencia-se a captação de mais turistas. Trata-se, pois, de uma atividade plural que coloca em equilíbrio direitos de cidadania e lucros (Kiefer \& Carvalho, 2013). Assim, neste turismo para Todos, também Todos são chamados a desenvolver experiências acessíveis e inclusivas numa perspetiva global e holística, no que se refere à conceção ou adaptação de serviços e equipamentos (Garcia, Papamichail \& Veitch, 2017).

Neste quadro, os serviços de alojamento detêm um papel de relevo no setor do turismo, uma vez que possuem a grande missão de acomodar quem está distante de casa. Com efeito, o alojamento é uma das principais prioridades para quem está a organizar uma viagem e, caso tenha algum tipo de necessidade específica, vai procurar um alojamento que responda a essa necessidade. Neste sentido, a adequação da oferta hoteleira deve respeitar o atendimento e a especificidade de todos os hóspedes, salientando-se, destarte, o Desenho Universal, uma vez que permite a criação de produtos e serviços de acesso fácil e autonónomo independentemente das características ou das limitações, tal como explica a Direção de Estudos e Planeamento Estratégico, do Turismo de Portugal (DEPE, 2012).

Neste trabalho, iremos abordar, num primeiro momento, a questão da acessibilidade nos espaços de alojamento, discutindo a importância da adaptação das infraestruturas e dos equipamentos, mas também dos serviços, numa lógica de consciencialização e eliminação dos diversos tipos de barreiras por parte da hotelaria como um todo (Kiefer \& Carvalho, 2013). Num segundo momento, iremos analisar uma das plataformas mais notórias em Portugal, a Turn4all, que oferece apoio às pessoas que procuram destinos turísticos e espaços de alojamento inclusivos. 


\section{Alojamento acessível e inclusivo: uma aposta de mercado?}

Parece-nos ter ficado claro que não se pode falar de turismo acessível sem que se faça referência aos espaços de alojamento. Com efeito, e tal como já foi aflorado anteriormente, também as pessoas com deficiência e com outras necessidades específicas são um público consumidor de produtos e serviços hoteleiros e, como tal, o mercado hoteleiro deve estar desperto para esta realidade e trabalhar o sentido da inclusão e acessibilidade, tendo como objetivo a eliminação das barreiras físicas e comportamentais, que impeçam ou dificultem a fruição dos produtos e serviços hoteleiros, bem como a garantia da competitividade de negócio (Kiefer \& Carvalho, 2013).

No entanto, esta questão de se pensar as infraestruturas realmente adaptadas a todos os cidadãos, quer tenham ou não necessidades específicas, vai muito para além da obrigação do cumprimento da lei, uma vez que a base de qualquer compromisso com a acessibilidade começa com a consciencialização para o tema.

Mediante o exposto, se é verdade que a grande maioria das pessoas vê de forma bastante positiva a promoção de espaços e serviços acessíveis a todos, os empresários do setor do turismo, designadamente do alojamento, precisam de perceber que a acessibilidade e o Desenho Acessível são oportunidades de negócio, que não devem ser desconsideradas (Garcia, Papamichail \& Veitch, 2017).

Também as Nações Unidas ${ }^{1}$ refletem sobre esta questão na sua página web, onde explicam que o turismo acessível engloba todos aqueles que detêm algum tipo de deficiência, mas também todos os que possam ter condicionalismos momentâneos bem como, todos os acompanhantes. Para além disso, há também a questão do envelhecimento que toca a todos. A bem dizer, a acessibilidade é um tema de tal modo transversal, que afeta todos os cidadãos e, ao dar resposta a Todos, tem um efeito multiplicador que aumenta a capacidade de negócio.

Estes benefícios empresariais começam a ser reconhecidos pelos gestores do setor, sendo também reforçados pelo próprio Turismo de Portugal (2013), através das várias inicitivas de divulgação e apoio à criação e reconversão dos espaços hoteleiros.

É oportuno dizer que em Portugal a consicência de que a acessibilidade é um mercado a explorar, porque é rentável, começa a ganhar peso e forma (Turismo de Portugal, 2016). Até há algum tempo, os benefícios empresariais do mercado de turismo acessível não eram muito conhecidos e, como tal, acabavam por ser desvalorizados, principalmente, porque se

\footnotetext{
1 https://www.un.org/development/desa/disabilities/issues/promoting-accessible-tourism-for-all.html
} 
acreditava que tornar os espaços acessíveis obrigava a grandes investimentos financeiros com um retorno muito baixo.

Contudo, os números mais recentes já demonstram que a aposta na acessibilidade dá origem a uma vantagem competitiva, que se consubstancia em maiores lucros, numa maior extensão da utilização da oferta turística, melhorando a competitividade, com evidentes benefícios para os agentes de turismo (Garcia, Papamichail \& Veitch, 2017; Direção de Estudos e Planeamento Estratégico, 2012). Deixemos os números falar por si: são cerca de 826 milhões de viagens na Europa que se estima serem realizadas por pessoas com necessidades específicas e "não menos importante é o número de visitantes portugueses com necessidades específicas - cerca de 634.400, um mercado que revela uma tendência cada vez maior para fazer day trips e short breaks, bem como férias mais prolongadas" (Garcia, Papamichail \& Veitch, 2017, pp. 9-10).

Já não estamos perante um nicho de mercado e esta ideia deve estar presente em todos os agentes de turismo, designadamente no setor do alojamento. Por isso, pensar as unidades de alojamento, tendo por base o Desenho Universal, não pode ser visto apenas como uma forma de responder às diretivas legais, mas mais como um investimento com retorno.

\subsection{Consciencializar os estabelecimentos de alojamento}

Em linha com o exposto até ao momento, já conseguimos perceber que o turismo acessível é um fenómeno em franco crescimento, onde o alojamento detém um papel especial. $\mathrm{O}$ turismo acessível e, logo, o alojamento acessível inclusivo, não se refere apenas à eliminação de barreiras físicas, mas também comportamentais, no tocante ao usufruto dos clientes, visando também assegurar a competitividade: "cresce a necessidade de consciencialização da atividade empresarial, no caso do turismo e hotelaria, sobre a importância de adequação a todos os segmentos do mercado" (Kiefer \& Carvalho, 2013, p. 1). Assim, e ainda segundo as mesmas autoras, "o treinamento dos funcionários dos meios de hospedagem e terceiros que prestam serviços em suas instalações são tão importantes quanto o oferecimento de espaços acessíveis/equipamentos/materiais específicos como material em Braille, telefones para pessoas com deficiência auditiva, rampas, banheiros adaptados e outros tantos" (Kiefer \& Carvalho, 2013, p. 2). Estamos a referir-nos, portanto, às várias dimensões da acessibilidade, que convocam muito mais do que a acessibilidade física.

A propósito deste assunto, Romeu Sassaki (2009) explica que a acessibilidade contempla seis dimensões: arquitetónica, comunicacional, metodológica, instrumental, programática e atitudinal. Assim, pensar em acessibilidade é ter em conta que se trata de uma componente que "desejamos ver e ter em todos os contextos e aspectos da atividade humana. Se a 
acessibilidade for (ou tiver sido) projetada sob os princípios do desenho universal, ela beneficia todas as pessoas, tenham ou não qualquer tipo de deficiência" (Sassaki, 2009, p. 2).

Esta questão, e este modo de pensar a acessibilidade, não tem passado despercebida no contexto mundial, onde Portugal não é exceção. O Estado Português, através do seu organismo Turismo de Portugal, tem tentado chegar junto dos empresários para que, em conjunto, contribuam para a inclusão de equipamentos mais acessíveis e capazes de acolher Todos. Neste desiderato, o Turismo de Portugal apresenta um conjunto de apoios e iniciativas que passam por questões financeiras, de formação e de parcerias inteligentes (Turismo de Portugal, 2016).

Sabe-se, também que perfil do consumidor mudou bastante nas últimas décadas. Hoje, o turista apresenta uma maior liberdade de escolha, tem a tendência de passar menos tempo em cada destino e procura uma maior interação com a população local, tal como explica o Serviço de Apoio às Micro e Pequenas Empresas do Estado de Pernambuco (SEBRAE, 2014). Esta realidade leva à necessidade de constantes adaptações, que assumem um papel de destaque nas agendas políticas portuguesas.

Conforme explicam Garcia, Papamichail e Veitch (2017, p. 49), desde 1997 que Portugal "estabeleceu como prioritário o combate às barreiras urbanísticas, arquitetónicas e comunicacionais, com uma lei sobre a acessibilidade: Decreto-lei 123/97 de 22 de maio, substituído pelo Decreto-Lei 163/2006, de 8 de agosto”. Foi criada também uma Estratégia Nacional para o Turismo (PENT revisão 2013-2015), que propôs um Plano de Ação para tornar Portugal um destino acessível a todos.

No ano de 2014 foi publicada a Norma Portuguesa NP 4523/2014 Turismo Acessível em Estabelecimentos Hoteleiros, assumindo-se enquanto referencial de qualidade na missão de apoiar estes estabelecimentos a destacarem-se pela prestação de serviços de turismo acessível, conforme se explica na página web do Turismo de Portugal $^{2}$. Assim, logo numa primeira instância, esta Norma apresenta como objetivo "promover boas práticas de serviço em estabelecimentos hoteleiros, com vista a definir um referencial de qualidade em matéria de atendimento inclusivo (da forma como os serviços são prestados) e acessibilidade do meio físico (das condições materiais para a prestação do serviço)", contribuindo para a facilitação do encontro entre a "oferta e a procura".

\footnotetext{
2 http://business.turismodeportugal.pt/pt/Gerir/reconhecimento-externo/normas-qualidade/Paginas/NP-4523-turismo-acessivel-em$\underline{\text { estabelecimentos-hoteleiros.aspx }}$
} 


\section{O exemplo da Turn4all}

Este casamento entre a oferta e a procura parece ter encontrado um veículo privilegiado em Portugal, a Turn4all ${ }^{3}$. Trata-se de uma app e de um website que permite ter dados sobre mais de 300 destinos e equipamentos turísticos, todos analisados por especialistas em acessibilidade ou avaliados e comentados pelos utilizadores. Nesta plataforma, os clientes são chamados a contribuir ativamente a partir das suas experiências nos locais que visitaram, ou onde estiveram hospedados. Para tal, podem utilizar uma grelha de avaliação (check list) já estruturada pela plataforma e, ainda, deixar os seus comentários. Esta grelha é transformada em relatório de avaliação e fica disponível junto ao equipamento hoteleiro, existindo, também, um espaço para os comentários dos utilizadores. Esta aposta nas perceções dos clientes é muito importante e só com o apoio (análise e comentários) de todos esta plataforma pode crescer, respostendo à melhoria da experiência turística. Pretende, pois, ser uma comunidade ativa de utilizadores, permitindo que a mesma avalie, pontue e comente a acessibilidade de todos os recursos turísticos. "Nada sobre nós, sem nós", lema das pessoas com deficiência, está bem plasmado nesta plataforma, onde o cliente tem uma voz ativa.

Continuando na análise da plataforma Tur4all, nomeadamente, a categoria alojamento, parece-nos importante perceber que informações compõem o relatório de cada equipamento hoteleiro, que parâmetros constituem a dita grelha de avaliação, bem como os comentários realizados pelos utilizadores. Uma análise global ao website, e aos equipamentos hoteleiros em particular, permite perceber que uma grande parte dos relatórios foram realizados por utilizadores, o que revela o envolvimento e a importância dos seus contributos nesta plataforma.

O relatório começa por apresentar o nome e a tipologia do equipamento, ilustado com uma fotografia do espaço exterior. Por baixo da fotografia, são indicados os contactos e apresentada a informação sobre a autoria do relatório, ou seja, se o mesmo foi preenchido por um técnico em acessibilidade ou por um utilizador. Os parâmetros preenchidos são os mesmos, podendo diferir um pouco no grau de especificidade que se atribui a um ou outro indicador.

Os parâmetros analisados são a acessibilidade física da entrada - se possui desnível e a largura da porta; a zona de atendimento ao público, designadamente o balcão de atendimento e os acessos; a circulação interior, mais concretamente o espaço para circulação com cadeira de rodas, a iluminação e a sinalética e o itinerário vertical (com

3 https://www.tur4all.pt/pt/tur4all/o-projeto-tur4all 
especial destaque para o elevador e escadas); quarto adaptado para limitação motora, que contempla aspetos como a circulação no interior do quarto e os espaços e produtos de apoio das casas de banho; áreas de restauração, outro parâmetro que, para além de contemplar as questões físicas (altura do balcão), também identifica outros indicadores como menu em caracteres ampliados e cor contrastante, opções para pessoas com alergias ou intolerâncias alimentares, serviço de mesa disponível, mesas redondas e cadeira para crianças; também está presente o parâmetro instalações sanitárias adaptadas nas zonas comuns; no parâmetro serviços e equipamentos é avaliado o staff de front-office, onde, em alguns relatórios, se pode ver uma avaliação à formação do pessoal, nomeadamente no atendimento a pessoas com necessidades específicas (apoio a pessoas com necessidades específicas e com formação em LGP). Contudo, este parâmetro encontra-se mais aprofundado, contemplando os indicadores enunciados, sobretudo nos relatórios elaborados pelos técnicos, sendo que na grande maioria dos relatórios realizados pelos utilizadores apenas é dada a indicação da existência de staff de front-office. Por fim, o relatório apresenta os seguintes parâmetros: estacionamento reservado PMR (para mobilidade reduzida) e espaço exterior, nomeadamente, passeio para peões e piso homogéneo e antiderrapante.

Assim, e mediante o exposto, parece-nos evidente que a acessibilidade física continua a ser a preocupação predominante quando se pensa em equipamentos hoteleiros inclusivos. Esta grelha, que é apresentada de uma forma muito prática e de fácil preenchimento, parece-nos esquecer outras dimensões da acessibilidade (nomeadamente a atitudinal e a comunicacional), que são tão importantes neste processo de superação de barreiras, prol à inclusão. Nesta equação, também os próprios utilizadores não se manifestam relativamente a essas outras dimensões. Com efeito, as dimensões que vão para além da física são mais exploradas pelos técnicos do que pelos utilizadores e os próprios espaços dedicados aos comentários estão praticamente todos por preencher, mesmo quando os relatórios são feitos pelos utilizadores, cinjindo-se, exclusivamente, aos parâmetros apresentados na grelha.

\section{Conclusões}

Em Portugal, existem diversos documentos orientadores e organismos que têm como missão impulsionar e apoiar o turismo acessível na superação de barreiras, rumo ao acesso e usufruto do lazer por Todos. Na realidade, em pleno século XXI, parece ser cada vez mais ponto assente que a acessibilidade "não é apenas uma ferramenta ou uma abordagem técnica [...] é um atributo cultural e atitudinal que engloba e enriquece todas as valências do atendimento ao cliente [...], contribuindo com vantagens adicionais para todos os negócios de turismo" (Garcia, Papamichail \& Veitch, 2017, p. 31). Neste quadro, falar de 
turismo acessível implica um compromisso com a acessibilidade em equipamentos hoteleiros, ou seja, alojamento acessível.

O alojamento acessível permite aos empresários ir ao encontro das necessidades das pessoas com deficiência, mas também daqueles que têm uma incapacidade temporária ou que vêm apenas como acompanhantes. Na verdade, vai ao encontro das necessidades de Todos e, por isso, é um atrativo de negócio. Porém, para que os alojamentos consigam ir efetivamente ao encontro das necessidades dos clientes, é importante que se consiga estabelecer a relação entre a "oferta e a procura", potenciando informações.

Para dar resposta a esta necessidade de mercado, foi criada a plataforma Tur4all que, assemelhando-se a um motor de busca, permite às pessoas que pretendem viajar perceber facilmente quais as condições de acessibilidade de destinos e equipamentos hoteleiros. As informações sobre a acessibilidade disponibilizadas pela plataforma advêm de técnicos especializados, mas, de igual modo, dos utilizadores. É uma plataforma colaborativa, onde os clientes do turismo acessível têm um papel importante.

Depois de analisarmos os relatórios disponibilizados relativamente aos diferentes equipamentos hoteleiros, verificámos que há uma atenção muio maior na dimensão física da acessibilidade, em detrimento de outras dimensões como a atitudinal ou comunicacional, que apenas são referidas ligeiramente. Este aspeto ainda é mais visível nos relatórios preenchidos pelos próprios utilizadores, que parecem não valorizar estas outras dimensões. Parece-nos que este aspeto pode estar associado ao facto haver uma maior regulamentação relativamente às condições físicas; contudo, para melhor se responder ao desafio da acessibilidade nos equipamentos de hotelaria, e em termos de estudos futuros, consideramos que seria interessante focar mais pormenorizadamente outros parâmetros de acessibilidade, nomeadamente atitudinal e comunicacional, pois entendemos que só esta visão mais holística permite um turismo verdadeiramente para Todos.

\section{Referências}

Beltramini, L. \& Júnior, A. (2018). Aplicação do desenho universal: uma análise da acessibilidade em hotéis de João Pessoa. In Atas do Congresso Luso-Brasileiro para o Planeamento Urbano, Regional, Integrado e Sustentável. Coimbra - Portugal, 24, 25 e 26 de outubro de 2018 (pp.1-13).

Direção de Estudos e Planeamento Estratégico (2012). Guia de Boas Práticas de Acessibilidade na Hotelaria. Lisboa: Turismo de Portugal. Retrieved from https://travelbi.turismodeportugal.pt/ptpt/Documents/Sustentabilidade/Boas $\% 20 \mathrm{Pr} \% \mathrm{C} 3 \% \mathrm{~A} 1$ ticas/guia-boas-praticas-acessibilidade$\underline{\text { hotelaria.pdf }}$

Garcia, A., Papamichail, K. \& Veitch, C. (2017). Manual de Gestão e de Destinos Turísticos Acessiveis. Lisboa: Turismo de Portugal. 
Sassaki, R. (2009). Inclusão: acessibilidade no lazer, trabalho e educação. Revista Nacional de Reabilitação (Reação), 2, 1-9.

Serviço de Apoio às Micro e Pequenas Empresas do Estado de Pernambuco - SEBRAE. (2014). Gostar de hospedar não é o bastante para empreender em hotelaria - Perfil de negócios de hotelaria (hotéis e pousadas). Recife: Sebrae/PE. Disponível em https:/www.sebrae.com.br/Sebrae/Portal\%20Sebrae/UFs/PE/Anexos/Perfil\%20de\%20negocios hotelaria .pdf

Turismo de Portugal (2013). Turismo Acessivel para Todos - Recomendações da OMT. Disponível $\mathrm{em}$ http://business.turismodeportugal.pt/SiteCollectionDocuments/all-for-all/recomendacoes-omtturismo-acessivel-para-todos.pdf

Turismo de Portugal (2016). Programa All for All - Portuguese Tourism. Disponível em http://business.turismodeportugal.pt/SiteCollectionDocuments/all-for-all/apresentacao-all-for-allset-2016.pdf

Turismo de Portugal (2017). Estratégia Turismo 2027 - Liderar o Turismo do Futuro. Disponível em http://institucional.turismodeportugal.pt/SiteCollectionDocuments/estrategia/estrategia-turismo2027.pdf 